\title{
A Theory for Log-Space and NLIN versus co-NLIN
}

\author{
Chris Pollett \\ 214 MacQuarrie Hall \\ Department of Computer Science \\ San Jose State University \\ 1 Washington Square, San Jose CA 95192 \\ pollett@cs.sjsu.edu
}

August 10, 2003-Draft

\begin{abstract}
The use of Nepomnjaščî̌'s Theorem in the proofs of independence results for bounded arithmetic theories is investigated. Using this result and similar ideas, it is shown that at least one of $S_{1}$ or $T L S$ does not prove the Matiyasevich-Robinson-Davis-Putnam Theorem. It is also established that $T L S$ does not prove a statement that roughly means nondeterministic linear time is equal to co-nondeterministic linear time. Here $S_{1}$ is a conservative extension of the well-studied theory $I \Delta_{0}$ and $T L S$ is a theory for LOGSPACE reasoning.
\end{abstract}

Mathematics Subject Classification: 03F30, 68Q15

Keywords: bounded arithmetic, independence results, MRDP

\section{Introduction}

In this paper applications of Nepomnjaščiř's Theorem to the provability of several important complexity statements in bounded arithmetic theories are considered. Recall that Nepomnjaščiǐ's Theorem states that those languages that can be decided in simultaneous time $n^{k}, k>0$ and space $n^{\epsilon}, 1>\epsilon$, the class $\operatorname{TISP}\left(n^{k}, n^{\epsilon}\right)$, are contained in the linear time hierarchy, LinH. The study of this theorem has recently undergone a renaissance since Fortnow [5] used it to prove time-space lower bounds for $S A T$.

The theory $I \Delta_{0}$ consists of defining axioms for the symbols of arithmetic together with induction for bounded formulas. By Wrathall [20] it is known that the $\Delta_{0}$-predicates in this language are the predicates computable in 
the linear time hierarchy, and so $I \Delta_{0}$ is in some sense a reasonable theory to reason about such sets. Numerous papers concerning how much number theory and combinatorics can be done in $I \Delta_{0}$ have been published and the interested reader should consult Hájek and Pudlák [7] or Krajíček [9] both as introductions to this area and for references into the literature.

Since Buss [1] presented a theory $S_{2}^{1}$ for polynomial time, many bounded arithmetic theories have been proposed to model reasoning about a variety of complexity classes. In particular, Clote and Takeuti [3] present theories for a variety of complexity classes within polynomial time. One such theory is TLS. Clote and Takeuti show that the essentially sharply bounded predicates of $T L S$ are precisely LOGSPACE. In a later paper [19], Takeuti shows that a subtheory of $T L S$ is able to prove the consistency of Frege propositional proof systems. From the point of view of propositional complexity Frege systems are considered quite strong and at the time of this writing no nontrivial lower bounds on proof size for families of tautologies in these systems are known. Cook [2] a describes a potentially stronger proof system still, L-Frege, and shows the second-order theory of Zambella [21] for LOGSPACE can prove $L$-Frege's consistency. It is quite likely that $T L S$ can also prove $L$-Frege's consistency.

The goal of this paper is to show that Nepomnjaščin's Theorem has important implications for the provable consequences of $I \Delta_{0}$ and $T L S$. The results are presented using a conservative extension of $I \Delta_{0}$ known as $S_{1}$ and a variant on Clote and Takeuti's TLS which is in a language with multiplication and is axiomatized in a simpler fashion than their theory. The version of TLS used here contains Clote and Takeuti's, still has as its $\hat{\Delta}_{1}^{b}$-predicates LOGSPACE. Using Nepomnjaščǐ's Theorem and Parikh's Theorem, it is shown that at least one of the theories $S_{1}$ and our $T L S$ cannot prove that all $\Sigma_{1}$-sets are Diophantine (i.e., the Matiyasevich-Robinson-Davis-Putnam (MRDP) Theorem [11]). It was already known that $I \Delta_{0}+\exp$, where exp is an axiom for exponentiation, proves the MRDP Theorem [6]. Being careful with how one defines a universal predicate for $\hat{\Sigma}_{i, k}^{\mathrm{b}}$-formulas, our paper also shows using Nepomnjaščiř's Theorem that TLS cannot prove $\hat{\Sigma}_{1,1}^{\mathrm{b}}=\hat{\Pi}_{1,1}^{\mathrm{b}}$. This is fairly close to saying (but not quite) that $T L S$ cannot prove NLIN =co-NLIN. Using the techniques of Pollett and Pruim [16], it is possible that the latter result could be obtained with the techniques of this paper but the expense would be to make TLS a more awkward looking theory. The arguments presented for the results above can be generalized to where simply defined functions of quasi-linear growth are added to both $T L S$ and $S_{1}$.

As a final point before proceeding to the outline of the paper, it should 
be noted that because of Parikh's Theorem, what the MRDP theorem is for a bounded arithemtic theory depends on the fastest growth rate functions in the underlying language. For instance, for $I \Delta_{0}$ to be able to prove MRDP, it suffices for it to show that linear sized bounded quantifiers can be eliminated in a Diophantine way. In the TLS case, since there is a function of growth rate $2^{|x||y|}$ in the language, one needs to be able to eliminate polynomial sized bounded quantifiers in a Diophantine way. Thus, the recent work in Pollett [15], which is in a language with $2^{x}$ is incomparable with the results of this paper.

This paper is organized as follows: The next section contains the notations and main definitions used in this paper. This is followed by a section showing that the $\hat{\Delta}_{1}^{b}$-predicates of $T L S$ are in fact LOGSPACE. The first two results listed in the abstract are then presented.

\section{Preliminaries}

The language $L_{1}$ contains the non-logical symbols: $0, S,+, \cdot, \leq,-,\left\lfloor\frac{1}{2} x\right\rfloor$, $|x|, \operatorname{PAD}(x, y)$, and $\operatorname{MSP}(x, i)$. The symbols $0, S(x)=x+1,+, \cdot$, and $\leq$ have the usual meaning. The intended meaning of $x-y$ is $x$ minus $y$ if this is greater than zero and zero otherwise, $\left\lfloor\frac{1}{2} x\right\rfloor$ is $x$ divided by 2 rounded down, and $|x|$ is $\left\lceil\log _{2}(x+1)\right\rceil$, that is, the length of $x$ in binary notation. $\operatorname{PAD}(x, y)$ is intended to mean $x \cdot 2^{|y|}$ and will be useful in defining a pairing function as an $L_{1}$-term. Finally, $\operatorname{MSP}(x, i)$ stands for 'most significant part' and is intended to mean $\left\lfloor x / 2^{i}\right\rfloor$. The language $L_{2}$ is $L_{1} \cup\{\#\} . x \# y$ reads ' $x$ smash $y$ ' and is intended to mean $2^{|x||y|}$. The notation 1 is used for $S(0)$, 2 for $S(S(0))$, etc. A quantifier of the form $(\forall x \leq t)$ or $(\exists x \leq t)$ where $t$ is a term not containing $x$ is called a bounded quantifier. A formula is bounded or $\Delta_{0}$ if all its quantifiers are. A quantifier of the form $(\forall x \leq|t|)$ or of the form $(\exists x \leq|t|)$ is called sharply bounded and a formula is sharply bounded if all its quantifiers are. Given a language $L$, the hierarchy of formulas $E_{i, L}$ and $U_{i, L}$ are defined as follows: $E_{1, L}$ are those formulas of the form $(\exists x \leq t) \phi$ and $U_{1, L}$ are those formulas of the form $(\forall x \leq t) \phi$ where $\phi$ is an open formula. $E_{i, L}$ are those formulas of the form $(\exists x \leq t) \phi$ where $\phi \in U_{i-1, L}$-formula. $U_{i, L}$ are those formulas of the form $(\forall x \leq t) \phi$ where $\phi \in E_{i-1, L}$. The notations $E_{i}$ and $U_{i}$ are used when $L$ is understood, and $E_{i, k}$ and $U_{i, k}$ are used for $E_{i, L_{k}}$ and $U_{i, L_{k}}$. The class of quantifier-free formulas is denoted by open (or open $_{k}$ to emphasize the language is $L_{k}$ ). For $i>0$, a $\hat{\Sigma}_{i}^{\mathrm{b}}$-formula (resp. $\hat{\Pi}_{i}^{\mathrm{b}}$-formula) is defined to be a $E_{i+1}$-formula (resp. $U_{i+1}$-formula) whose innermost quantifier is sharply bounded. To emphasize the language 
is $L_{k}$ we write $\hat{\Sigma}_{i, k}^{\mathrm{b}}$ and $\hat{\Pi}_{i, k}^{\mathrm{b}}$. The classes $\Sigma_{i}^{\mathrm{b}}$ and $\Pi_{i}^{\mathrm{b}}$ are the closures of $\hat{\Sigma}_{i}^{\mathrm{b}}$ and $\hat{\Pi}_{i}^{\mathrm{b}}$ under subformulas, $\wedge, \vee$, and sharply bounded quantifications. Kent and Hodgson [8] (see also Pollett [17]) have shown the sets defined by $\hat{\Sigma}_{i, 2^{-}}^{\mathrm{b}}$ (resp. $\hat{\Pi}_{i, 2^{-}}^{\mathrm{b}}$ )formulas are precisely the $\Sigma_{i}^{p}$-(resp. $\Pi_{i}^{p}$-)predicates. Thus, the $\hat{\Sigma}_{1,2}^{b}$-formulas correspond to the NP-predicates.

The theory $B A S I C_{k}$ is axiomatized by all substitution instances of a finite set of quantifier free axioms for the non-logical symbols of $L_{k}, k=1,2$. These are listed in Buss [1] except for the axioms for MSP and - which are listed in Takeuti [18], and those for PAD are listed in Clote and Takeuti [3].

For this paper, it is useful to be able to have a pairing function, as well as to have functions that can project blocks of bits from a number so that a limited amount of sequence coding can be done. These can be defined using $L_{1}$-terms as follows: For projection of bits, define the functions $2^{|y|}:=\operatorname{PAD}(1, y), 2^{\min (|y|, x)}:=\operatorname{MSP}\left(2^{|y|},|y|-x\right), \operatorname{LSP}(x, i):=x-\operatorname{MSP}(x, i)$. $2^{\min (|x|, i)}, \hat{\beta}_{|t|}(x, w):=\operatorname{MSP}(\operatorname{LSP}(w,(S x)|t|), x|t|)$, and $\operatorname{BIT}(i, x):=\hat{\beta}_{1}(i, x)$. Here $\hat{\beta}$ is supposed to project the $x$ th block of $|t|$ bits from $w$ and BIT is supposed to return the $i$ th bit of $x$. Given these functions to define pairing operations, let $\max (x, y):=(1-((x+1)-y))) y+(1-(y-x)) x$ and set $B=2^{|\max (x, y)|+1}$. Thus, $B$ will be longer than either $x$ or $y$. Define an ordered pair as $\langle x, y\rangle:=\left(2^{|\max (x, y)|}+y\right) \cdot B+\left(2^{|\max (x, y)|}+x\right)$. To project out the coordinates from such an ordered pair, use $(w)_{1}:=\hat{\beta}_{\left\lfloor\frac{1}{2}|w|\right\rfloor-1}\left(0, \hat{\beta}_{\left\lfloor\frac{1}{2}|w|\right\rfloor}(0, w)\right)$ and $(w)_{2}:=\hat{\beta}_{\left\lfloor\frac{1}{2}|w|\right\rfloor-1}\left(0, \hat{\beta}_{\left\lfloor\frac{1}{2}|w|\right\rfloor}(1, w)\right)$ which return the left and right coordinates of the pair $w$. To check if $w$ is a pair the formula $i \operatorname{spair}(w):=$

$$
\operatorname{Bit}\left(w,\left\lfloor\frac{1}{2}|w|\right\rfloor-1\right)=1 \wedge 2 \cdot\left|\max \left((w)_{1},(w)_{2}\right)\right|+2=|w|
$$

is used. The usual properties of this formula as well as the terms listed above are provable in the theories we will consider in this paper [17].

The theories in this paper will all be formulated in the sequent calculus system $L K B$ of Buss [1].

Definition $1 A \Psi-L^{m} I N D$ inference is an inference:

$$
\frac{A(b), \Gamma \rightarrow A(S b), \Delta}{A(0), \Gamma \rightarrow A\left(|t(x)|_{m}\right), \Delta}
$$

where $b$ is an eigenvariable and must not appear in the lower sequent, $t \in L_{2}$, $|x|_{0}=x$, and $|x|_{m+1}=\left.|| x\right|_{m} \mid$.

The notations IND, LIND, LLIND will be used instead of $L^{0} I N D$, $L^{1} I N D$, and $L^{2} I N D$. 
Definition $2(i \geq 0)$ The theories $T_{k}^{i}$ and $S_{k}^{i}$ are $B A S I C_{k}+\hat{\Sigma}_{i, k}^{\mathrm{b}}-I N D$ and $B A S I C_{k}+\hat{\Sigma}_{i, k}^{\mathrm{b}}-L I N D$, respectively.

We define $S_{k}:=\cup_{i} S_{k}^{i}$.

That $S_{k}^{i}$ and $T_{k}^{i}$ can be equivalently defined using $\hat{\Sigma}_{i, k}^{\mathrm{b}}$ induction schemas rather than $\Sigma_{i, k}^{\mathrm{b}}$ schemas was shown in Pollett [17]. From Buss [1] is it known that

$$
S_{k}^{i} \subseteq T_{k}^{i} \subseteq S_{k}^{i+1}
$$

The theory $I \Delta_{0}$ is defined using the language $0, S,+, \cdot ; \leq$. It consists of axioms for these symbols together with $\Delta_{0}-I N D$. The symbols in $L_{1}$ are all definable in $I \Delta_{0}$, and it is known that $S_{1}$ is a conservative extension of $I \Delta_{0}$. For more details on this relationship and this theory, the reader is advised to consult Krajíček [9].

The last definitions needed to present $T L S$ are now given.

Definition 3 Given a term $t$ in one of the languages of this paper we define a monotonic term $t^{*}$ as follows: If $t$ is constant or a variable, then $t=t^{*}$. If $t$ is $f(s)$, where $f$ is a unary function symbol, then $t^{*}$ is $f\left(s^{*}\right)$. If $t$ is $s_{1} \circ s_{2}$ for $\circ$ a binary operation other than - or $M S P$, then $t^{*}$ is $s_{1}^{*} \circ s_{2}^{*}$. Lastly, if $t$ is $s_{1}-s_{2}$ or $\operatorname{MSP}\left(s_{1}, s_{2}\right)$, then $t^{*}$ is $s_{1}^{*}$.

It is easily proved in BASIC+open-LIND that $t^{*}$ is monotonic, and $t \leq t^{*}$.

In the next definition, $\exists$ ! is used to abbreviate two sequents expressing uniqueness and existence.

Definition 4 The $\Psi$-WSN (weak successive nomination rule) is the following rule:

$$
\frac{b \leq|k(j, \vec{a})| \rightarrow \exists ! x \leq|k| A(j, \vec{a}, b, x)}{\rightarrow \exists w \leq \operatorname{bd}(|k|, t) \forall j<|t| A\left(j, \vec{a}, \hat{\beta}_{\left|k^{*}\right|}(j, w), \hat{\beta}_{\left|k^{*}\right|}(S j, w)\right)}
$$

where $A \in \Psi$ and $\operatorname{bd}(a, b):=2(2 a \# 2 b)$.

The last rule needed to define $T L S$ is:

Definition $5 \Psi$-REPL (quantifier replacement) is the following rule:

$$
\begin{aligned}
& (\forall x \leq|s|)(\exists y \leq t(x, a)) A(x, y, a) \Leftrightarrow \\
& \quad\left(\exists w \leq \operatorname{bd}\left(t^{*}(|s|, a), s\right)\right)(\forall x \leq|s|) A\left(x, \dot{\beta}_{\left|t^{*}(|s|, a)\right|, t}(x, w)\right)
\end{aligned}
$$

where $A \in \Psi$ and $\dot{\beta}_{t, s}(x, w):=\min \left(\hat{\beta}_{t}(x, w), s\right)$. Here $\min (x, y):=x+y-$ $\max (x, y)$.

Definition 6 TLS is BASIC ${ }_{2}+$ open $_{2}-L I N D+\hat{\Sigma}_{1,2}^{\mathrm{b}}-W S N+\hat{\Sigma}_{1,2}^{\mathrm{b}}-R E P L$. 


\section{Bootstrapping}

$T L S$ is axiomatized in a different fashion than the version presented in Clote and Takeuti [3]. The theory here actually has a slightly stronger axiomatization. Nevertheless, in this section it is argued that its $\hat{\Delta}_{1}^{b}$-predicates are still LOGSPACE.

Recall $A$ is said to be $\hat{\Delta}_{i}^{b}$ in a theory $T$ if $T \vdash A^{\Sigma} \equiv A \equiv A^{\Pi}$ where $A^{\Sigma}$ is $\hat{\Sigma}_{i}^{\mathrm{b}}$ and $A^{\Pi}$ is $\hat{\Pi}_{i}^{\mathrm{b}}$. $\Delta_{i}^{b}$ is defined analogously, but using $\Sigma_{i}^{\mathrm{b}}$ and $\Pi_{i}^{\mathrm{b}}$. Recall also that $f$ is $\Psi$-defined in $T$ if there is a $\Psi$-formula $A$ such that $\mathbb{N} \models A(x, f(x))$ and $T \vdash \forall x \exists ! y A(x, y)$. Because $T L S$ proves quantifier replacement for $\hat{\Sigma}_{1}^{\mathrm{b}}$-formulas, the notions of $\Sigma_{1}^{\mathrm{b}}$-definability and $\hat{\Sigma}_{1}^{\mathrm{b}}$-definability coincide; similarly, the notions $\hat{\Delta}_{1}^{b}$ and $\Delta_{1}^{b}$-coincide.

Johannsen and Pollett [10] give two theories for the $\mathrm{TC}^{0}$-predicates (predicates computable by constant depth threshold circuits), $C_{2}^{0}$ and $\Delta_{1}^{b}-C R$. The former theory is of interest in the present discussion. It was axiomatized as BASIC+open $2_{2}-L I N D$ and $\Sigma_{0}^{\mathrm{b}}-R E P L$ and so is contained in TLS. This is because it is easy to show $\Sigma_{0}^{\mathrm{b}}-R E P L$ and in fact even $\Sigma_{1}^{\mathrm{b}}-R E P L$ using $\hat{\Sigma}_{1}^{\mathrm{b}}-R E P L$. Given that $\Sigma_{0}^{\mathrm{b}}-R E P L$ implies $\Sigma_{1}^{\mathrm{b}}-R E P L$ by the same method as was used in Buss [1] to show $\Pi_{i}^{\mathrm{b}}-R E P L$ implies $\Sigma_{i+1}^{\mathrm{b}}-R E P L$, the only difference between $C_{2}^{0}$ and $T L S$ is that the latter theory has $\hat{\Sigma}_{1,2^{-}}^{b} W S N$. In what follows, a function is said to be in $\mathrm{TC}^{0}$ or in LOGSPACE, if its graph is in the given class and if the number of bits in its output is polynomial in the number of input bits.

Theorem 1 (1) The $\hat{\Sigma}_{1}^{\mathrm{b}}$-definable functions of TLS are exactly LOGSPACE. (2) The $\hat{\Delta}_{1}^{b}$-predicates of TLS are exactly the LOGSPACE predicates.

Proof. From Clote and Takeuti [3], the functions in LOGSPACE can be viewed as the closure $\mathrm{TC}^{0}$ under $B_{2} R N$. Here a function $f$ is defined by $B_{2} R N$ from the functions $g, h_{0}, h_{1}$, and $k$ if $f(0, \vec{x})=g(\vec{x})$, $f(2 n, \vec{x})=h_{0}(n, \vec{x}, f(n, \vec{x})), f(2 n+1, \vec{x})=h_{1}(n, \vec{x}, f(n, \vec{x}))$ and, in addition, it is required that $f(n, \vec{x})<|k(n, \vec{x})|$. Given that TLS contains $C_{2}^{O}$ and Johannsen and Pollett [10] show the $\Sigma_{1}^{\mathrm{b}}$-definable functions of $C_{2}^{0}$ are precisely $\mathrm{TC}^{0}$, it follows $T L S$ can $\Sigma_{1}^{\mathrm{b}}$-define $\mathrm{TC}^{0}$. Using $\hat{\Sigma}_{1}^{\mathrm{b}}-R E P L$, it can thus $\hat{\Sigma}_{1}^{\mathrm{b}}$-define these functions. Then by using $\hat{\Sigma}_{1}^{\mathrm{b}}$ WSN and essentially same argument as used in Theorem 5.1 and 6.3 by Clote and Takeuti [3] for their version of $T L S$, one can show $T L S$ can proves it $\hat{\Sigma}_{1}^{\mathrm{b}}$-definable functions closed under $B_{2} R N$. For the other direction, one needs to carry out a Buss-style witnessing argument, to show that only the LOGSPACE functions are $\hat{\Sigma}_{1}^{\mathrm{b}}$-definable by $T L S$. This argument is essentially the same as 
the witnessing argument of Johannsen and Pollett [10] to show $C_{2}^{0}$ can only $\hat{\Sigma}_{1}^{b}$-define $\mathrm{TC}^{0}$ functions. The only additional case is to handle $\hat{\Sigma}_{1}^{\mathrm{b}}-W S N$. The witness function in this case is constructed using $B_{2} R N$ in a similar fashion to Theorem 5.2 of Clote and Takeuti. The reader interested in more of the gory details can consult the technical report Pollett [14]. Given that the $\hat{\Sigma}_{1}^{\mathrm{b}}$-definable functions of $T L S$ are those functions in LOGSPACE, the fact that the $\hat{\Delta}_{1}^{b}$-predicates of TLS are exactly LOGSPACE, follows from the usual correspondence between $0-1$ valued $\hat{\Sigma}_{1}^{\mathrm{b}}$-definable functions and the $\hat{\Delta}_{1}^{b}$-predicates of a theory. This argument can be found in Buss [1].

\section{Independence results}

To begin some well known results are recalled:

Theorem 2 (1) The predicates in $\cup_{i} \hat{\Sigma}_{i, 1}^{\mathrm{b}}$ are precisely $\mathrm{LinH}$. (Wrathall [20]) (2) For $i>0, \hat{\Sigma}_{i, 2}^{\mathrm{b}}=\Sigma_{i}^{\mathrm{p}}$.(Kent-Hodgson [8])

Theorem 3 (Nepomnjaščǐ [12]) LinH contains TISP $\left(n^{k}, n^{1-\epsilon}\right)$. So LinH contains LOGSPACE.

The next lemma provides a universal predicate for $\hat{\Sigma}_{i}^{\mathrm{b}}$-formulas which will be convenient to work with in the sequel.

Lemma 1 There is a $\hat{\Sigma}_{i, 1}^{\mathrm{b}}$-formula (note the 1$), U_{i}(e, x, z)$, such that for any $\hat{\Sigma}_{i, 2}^{\mathrm{b}}$-formula $A(x)$ there is a number $e_{A}$ and $L_{2}$-term $t_{A}$ for which

$$
T L S \vdash U_{i}\left(e_{A}, x, t_{A}(x)\right) \equiv A(x) .
$$

If $A$ is in $\hat{\Sigma}_{i, 1}^{\mathrm{b}}$ then $t_{A}$ can be chosen to be an $L_{1}$-term in $x$ or we can choose a single $L_{2}$-term $t\left(e_{A}, x\right)$ which works for all $A$.

Proof. Using $\mathrm{K}_{\neg}(x):=1-x, \mathrm{~K}_{\vee}(x, y):=x+y$, and $\mathrm{K}_{\leq}(x, y):=\mathrm{K}_{\neg}(y \dot{-} x)$, one can write any open formula $A(x, \vec{y})$ as an equation $f(x, \vec{y})=0$ where $f \in L_{k}$. By induction, on the complexity of $A$ this is provable in $T L S$. So any $\hat{\Sigma}_{i}^{\mathrm{b}}$-formula $\phi(x)$ is provably equivalent in $T L S$ to one of the form

$$
\left(\exists y_{1} \leq t_{1}\right) \cdots\left(Q y_{i} \leq t_{i}\right)\left(Q^{\prime} y_{i+1} \leq\left|t_{i+1}\right|\right)\left(t_{i+2}(x, \vec{y})=0\right)
$$

where the quantifiers $Q$ and $Q^{\prime}$ will depend on whether $i$ is even or odd. We fix some coding scheme for the 12 symbols of $L_{2}$ as well as for the $i+2$ variables $x, y_{1}, \ldots, y_{i+1}$. We use $\Pi$ to denote the code for some symbol. i.e., 
$\lceil=1$ is the code for $=$. We choose our coding so that all codes require less than $|i+14|$ bits and 0 is used as $\left.{ }^{\lceil N O P}\right\rceil$ meaning no operation. Thus, if one tries to project out operations beyond the end of the code of the term one naturally just projects out $\left\lceil^{\top N O P}\right]^{\prime}$ 's. The code for a term $t$ is a sequence of blocks of length $|i+14|$ that write out $t$ in postfix order. So $x+y_{1}$ would be coded as the three blocks $\left\lceil_{x}\right\rceil\left\lceil_{1}\right\rceil\lceil+\rceil$. The code for a $\hat{\Sigma}_{i}^{\mathrm{b}}$-formula will be $\left.\left.\left\langle\left\langle t_{1}\right\rceil, \ldots,{ }_{t i+3}\right\rceil\right\rangle\right\rangle$. We now describe $U_{i}(e, x, z)$. It will be obtained from the formula

$$
\begin{gathered}
(\exists w \leq z)\left(\exists y_{1} \leq z\right)(\forall j \leq|e|)\left(\forall y_{2} \leq z\right) \cdots \\
\cdots\left(Q y_{i} \leq z\right)\left(Q^{\prime} y_{i+1} \leq|z|\right) \phi_{i}(e, j, x, \vec{y})
\end{gathered}
$$

after pairing is applied. Here $\phi_{i}$ consists of a statement saying $w$ is a tuple of the form $\left\langle\left\langle w_{1}, \ldots, w_{i+2}\right\rangle\right\rangle$ together with statements saying each $w_{i}$ codes a postfix computation of $t_{i}$ in $\left.\left.e=\left\langle\left\langle{ } t_{1}\right\rceil, \ldots,{ }_{t_{i+3}}\right\rceil\right\rangle\right\rangle$. If $z^{\prime}:=\operatorname{MSP}\left(z,\left\lfloor\frac{1}{2}|z|\right\rfloor\right)$ (roughly, the square root of $z$ ) is used as the block size, this amounts to checking conditions for each $m$

$$
\begin{aligned}
& {\left[\hat{\beta}_{|i+14|}\left(j,{ }_{t_{m}}{ }^{\top}\right)=\left\lceil^{\rceil}\right\rceil \supset \hat{\beta}_{\left|z^{\prime}\right|}\left(j, w_{m}\right)=x\right] \wedge} \\
& {\left[\hat{\beta}_{|i+14|}\left(j,{ }^{t_{m}}{ }^{\top}\right)=\lceil+\rceil \supset\right.} \\
& \left.\hat{\beta}_{\left|z^{\prime}\right|}\left(j, w_{m}\right)=\hat{\beta}_{\left|z^{\prime}\right|}\left(j-2, w_{m}\right)+\hat{\beta}_{\left|z^{\prime}\right|}\left(j-1, w_{m}\right)\right] \wedge \cdots \\
& {\left[\hat{\beta}_{|i+14|}\left(j, \Gamma_{t_{m}}{ }^{\top}\right)=\lceil \#\rceil \supset\right.} \\
& \left|\hat{\beta}_{\left|z^{\prime}\right|}\left(j, w_{m}\right)\right|=S\left(\left|\hat{\beta}_{\left|z^{\prime}\right|}\left(j-2, w_{m}\right)\right|\left|\hat{\beta}_{\left|z^{\prime}\right|}\left(j-1, w_{m}\right)\right|\right) \\
& \left.\wedge \operatorname{LSP}\left(\hat{\beta}_{\left|z^{\prime}\right|}\left(j, w_{m}\right),\left|\hat{\beta}_{\left|z^{\prime}\right|}\left(j, w_{m}\right)\right|-1\right)=0\right] \wedge \cdots \\
& {\left[\hat{\beta}_{|i+14|}\left(j,\left\ulcorner_{t_{m}}{ }^{\top}\right)={ }^{\top N O P}\right\rceil \supset \hat{\beta}_{\left|z^{\prime}\right|}\left(j, w_{m}\right)=\hat{\beta}_{\left|z^{\prime}\right|}\left(j-1, w_{m}\right)\right] .}
\end{aligned}
$$

$\phi_{i}$ also has conditions $y_{m} \leq \hat{\beta}_{\left|z^{\prime}\right|}\left(|e|, w_{m}\right) \wedge$ if $y_{m}$ was existentially quantified and conditions $y_{m} \leq \hat{\beta}_{\left|z^{\prime}\right|}\left(|e|, w_{m}\right) \supset$ if $y_{m}$ was universally quantified. Notice none of the conditions above make use of the \# function. Finally, $\phi_{i}$ has a condition saying $\hat{\beta}_{\left|z^{\prime}\right|}\left(|e|, w_{i+2}\right)=0$. Since $T L S$ can prove simple facts about projections from pairs, it can prove by induction on the complexity of the terms in any $\hat{\Sigma}_{i}^{\mathrm{b}}$-formula $\phi(x)$ that $U_{i}\left(e_{\phi}, x, t\left(e_{\phi}, x\right)\right) \equiv \phi(x)$ provided $t\left(e_{\phi}, x\right)$ is large enough.

To estimate the size of $t_{A}$, an upper bound on $w_{m}$ is calculated. First, all real formulas $A$ have their terms represented as trees, so we can assume $e_{A}$ codes terms which are trees. By induction over the subtrees of a given term 
$t_{m}$, one can show an upper bound on the block size needed to store a step of $w_{m}$ of the form $\left|e_{m}\right|\left(|x|+\left|e_{A}\right|\right)$. So the length of any $w_{m}$ can be bounded by $\ell=\left|e_{A}\right|\left|e_{A}\right|\left(|x|+\left|e_{A}\right|\right)>\left|e_{m}\right|\left|e_{m}\right|\left(|x|+\left|e_{A}\right|\right)$. So choosing an $L_{1}$-term larger than $2^{(i+2) \ell}$ suffices. This is possible since $e_{A}$ is a fixed number. Notice if both $e_{A}$ and $x$ are viewed as parameters, this is in fact boundable by an $L_{2}$-term $t$. If $A$ does involve \# than a similar estimate can be done to show that an $L_{2}$-term for $t_{A}$ suffices.

Lemma 2 For $i \geq 1, \hat{\Sigma}_{i, 1}^{\mathrm{b}} \neq \hat{\Pi}_{i, 2}^{\mathrm{b}}$.

Proof. If $A$ is in $\hat{\Sigma}_{i, 1}^{\mathrm{b}}$ then the last argument of $U_{i}$ from Lemma 1 is an $L_{2}$-term. So there is a $\hat{\Sigma}_{i, 2}^{\mathrm{b}}$-formula $U\left(x, e_{A}\right) \equiv A$ for all $A$ in $\hat{\Sigma}_{i, 1}^{\mathrm{b}}$. Consider $\neg U(x, x)$ this formula is equivalent to a $\hat{\Pi}_{i, 2}^{\mathrm{b}}$-formula. Also, it is easy to see it is not in $\hat{\Sigma}_{i, 1}^{\mathrm{b}}$.

The independence results in this section are all a consequence of the following lemma:

Lemma 3 If $\hat{\Sigma}_{i, 1}^{\mathrm{b}}=\hat{\Pi}_{i, 1}^{\mathrm{b}}$ then LOGSPACE $\neq N \mathrm{NP}$.

Proof. $\quad$ Suppose $\hat{\Sigma}_{i, 1}^{\mathrm{b}}=\hat{\Pi}_{i, 1}^{\mathrm{b}}$ and LOGSPACE $=$ NP. As LOGSPACE is closed under complement LOGSPACE $=\mathrm{PH}$. By Theorem 3 and Theorem 2 LOGSPACE is contained in $\mathrm{LinH}$, and we have that $\hat{\Sigma}_{i, 1}^{\mathrm{b}}=\mathrm{LinH}=\mathrm{PH}$. But by Lemma 2 , there are languages in $\hat{\Pi}_{i, 2}^{\mathrm{b}}$ that are not in $\hat{\Sigma}_{i, 1}^{\mathrm{b}}$.

Lemma 3 is similar to a result of Ferreira [4] where it is shown that LOGSPACE $=\Delta_{0}$ implies $\Delta_{0} \not \subset \Sigma_{s}^{l}$. Here $\Sigma_{s}^{l}$ is a secord-order class of formulas defining sets similar to $\Sigma_{s, 1}^{\mathrm{b}}$. Ferreira's argument was model theoretic. One consequence of Lemma 3 concerns the provability of the MatiyasevichRobinson-Davis-Putnam (MRDP) Theorem [11] in bounded arithmetic. Recall the MRDP Theorem says that the $\Sigma_{1}$-sets are equivalent to the sets that can be defined by formulas of the form:

$$
A=\{x \mid(\exists \vec{y}) P(x, \vec{y})=Q(x, \vec{y})\},
$$

where $P, Q$ are polynomials with coefficients in $\mathbb{N}$. It is known that $I \Delta_{0}+e x p$, where exp is an axiom for exponentiation, proves the MRDP Theorem [6]. To prove our result, we first have need of a well-known lemma whose proof we include for completeness.

Lemma 4 Let $T$ be one of $S_{k}^{i}, S_{k}$ or TLS. If T proves the MRDP theorem then $T$ proves $E_{1, k}=U_{1, k}$. 
Proof. To see this, suppose $T$ proves the MRDP theorem. Then for every $U_{1, k}$-formula $A(\vec{x})$ there is a formula $F(\vec{x}):=(\exists \vec{y}) P(\vec{x}, \vec{y})=Q(\vec{x}, \vec{y})$ where $P, Q$ are polynomials such that $T \vdash A \equiv F$. In particular, $T$ proves $A \rightarrow(\exists \vec{y}) P(\vec{x}, \vec{y})=Q(\vec{x}, \vec{y})$. By Parikh's theorem (see Hájek and Pudlák [7] for a proof), since $T$ is a bounded theory one can bound the $\vec{y}$ 's by an $L_{k^{-}}$ term $t$ giving an $E_{1, k}$-formula $F_{2}$. Note $F_{2} \supset F \supset A$ so $A \equiv F_{2}$ completing the proof.

Theorem 4 At least one of $S_{1}$ and TLS does not prove MRDP.

Proof. By the previous lemma, if $S_{1}$ proves the MRDP Theorem then $\mathrm{LinH}=\hat{\Sigma}_{1,1}^{\mathrm{b}}$. By a similar, argument if $T L S$ proves MRDP Theorem then LOGSPACE $=\hat{\Pi}_{1,2}^{\mathrm{b}}=\hat{\Sigma}_{1,2}^{\mathrm{b}}=\mathrm{PH}$. Thus, we contradict Lemma 3.

The next theorem gives another application of Lemma 3 .

Theorem 5 TLS cannot prove $\hat{\Sigma}_{1,1}^{b}=\hat{\Pi}_{1,1}^{b}$.

Proof. Suppose TLS proves $\hat{\Sigma}_{1,1}^{\mathrm{b}}=\hat{\Pi}_{1,1}^{\mathrm{b}}$. This means that for each $\hat{\Sigma}_{1,1^{-}}^{\mathrm{b}}$ formula $A$ we can find some $\hat{\Pi}_{1,1}^{\mathrm{b}}$-formula $B$ such that $T L S \vdash A \equiv B$. Let $A(x):=\exists y \leq t(x) D(x, y)$ be an arbitrary $\hat{\Sigma}_{1,2}^{\mathrm{b}}$-formula in one variable. Let $C(x, z):=U_{1}\left(e_{A}, x, z\right)$ where $U_{1}$ is from Lemma 1 . So $C$ is a $\hat{\Sigma}_{1,1}^{\mathrm{b}}$-formula, and, thus, by assumption, provably equivalent to some $\hat{\Pi}_{1,1}^{\mathrm{b}}$-formula $C^{\prime}(x, z)$ in $T L S$. So $T L S$ proves

$$
A \equiv C\left(x, t_{A}(x)\right) \equiv C^{\prime}\left(x, t_{A}(x)\right)
$$

where $t_{A}$ is the bounding term on $U_{1}$ in Lemma 1 . The last formula is a $\hat{\Pi}_{1,2}^{\mathrm{b}}$-formula. Hence, it follows that $T L S$ proves $\hat{\Sigma}_{1,2}^{\mathrm{b}}=\hat{\Pi}_{1,2}^{\mathrm{b}}$. i.e., NP $=$ coNP. As the $\hat{\Delta}_{1}^{b}$-formulas of TLS are LOGSPACE, one also gets that $\hat{\Sigma}_{1,2}^{\mathrm{b}}=$ LOGSPACE. But this contradicts Lemma 3.

Remark 1 The results presented above are reasonably insensitive to the underlying language as long as the functions symbols added are LOGSPACE computable and have $O\left(n^{1+o(1)}\right)$ growth rate. For instance, one could add to $L_{1}$ and $L_{2}$ a symbol for $x \#|y|$ and add to $S_{1}$ and TLS defining axioms for this symbol. The resulting TLS would be conservative over the TLS used above. On the hand, the $\Delta_{0}$-sets in the resulting $L_{1}$ would now define the quasilinear time hierarchy and the resulting $S_{1}$ would be able to reason about such sets. Nevertheless, the part of Lemma 1 concerning a single $L_{2}$-term able to 
work for all $A$ still holds. Now, though, a bound on the length of the code for computation of $e_{A}$ will be $2^{(i+2) \ell}$ where $\ell$ is $O\left(\left(|x|+\left|e_{A}\right|\right)\left(|| x|+| e_{A}||\right)^{\left|e_{A}\right|}\right)$. If one requires that $e_{A} \leq\|x\|$ then strings of this length can be bounded by an $L_{1}$-term. So in Lemma 2, one now considers a $\hat{\Pi}_{1,2}^{\mathrm{b}}$ predicate $\neg U(x,\|x\|)$ to diagonalize out of $\hat{\Sigma}_{1,1}^{\mathrm{b}}$. All the other results of this section also hold. Hence, it still holds that at least one of $S_{1}$ or TLS in the new languages does not prove MRDP and also that TLS does not prove $\hat{\Sigma}_{1,1}^{\mathrm{b}}=\hat{\Pi}_{1,1}^{\mathrm{b}}$.

\section{Conclusion}

Hájek and Pudlák [7] develop definitions for context free grammars in the theory $I \Delta_{0}$. Thus, it is quite likely that the results of this paper could be extended to a theory whose $\Delta_{1}^{b}$-predicates were LOGCFL. Here LOGCFL is the class of languages logspace reducible to context free languages. It is known that LOGCFL contains NLOGSPACE. So such a result seems like the next logical step in pushing the techniques of this paper.

\section{References}

[1] S.R. Buss. Bounded Arithmetic. Bibliopolis, Napoli, 1986.

[2] S. Cook. A Survey of Complexity Classes and their Associated Propositional Proof Systems and Theories, and a Proof System for Log Space Slides for Edinburgh talk, presented at the ICMS Workshop "Circuit and Proof Complexity", Edinburgh, October, 2001.

[3] P. Clote and G. Takeuti. First order bounded arithmetic and small boolean circuit complexity classes. In P. Clote and J. Remmel, editors, Feasible Mathematics II, pages 154-218. Birkhäuser, Boston, 1995.

[4] F. Ferreira. A proof that LOGSPACE $\neq$ NLIN. Unpublished notes. 1998.

[5] L. Fortnow Time-space tradeoffs for satisfiability. Journal of Computer and System Sciences, 60(2):337-353, April 2000.

[6] H. Gaifman and C. Dimitracopoulos. Fragments of Peano's arithmetic and the MRDP theorem. Monographie 30 de L'Enseignement Mathématique, pages 187-206, 1982.

[7] P. Hájek and P. Pudlák. Metamathematics of First-Order Arithmetics. Springer-Verlag, 1993. 
[8] C. F. Kent and B.R. Hodgson. An arithmetical characterization of NP. Theoretical Computer Science, 21:255-267, 1982.

[9] J. Krajíček. Bounded Arithmetic, Propositional Logic and Complexity Theory. Cambridge University Press, 1995.

[10] J. Johannsen and C. Pollett. On the $\Delta_{1}^{b}$-Comprehension Rule. In S. Buss, P. Hájek and P. Pudlák Lecture Notes in Logic 13 - Logic Colloquium 1998, pages 269-286, A.K. Peters, 2000.

[11] Y. Matiyasevich. Enumerable sets are Diophantine. Dokl. Acad. Nauk, 191:279-282, 1970.

[12] V.A. Nepomnjaščii. Rudimentary predicates and Turing computations. Dokl. Acad. Nauk, Vol. 195, pages 282-284, 1970, transl. Vol. 11 14621465, 1970.

[13] C. Pollett. Multifunction algebras and the provability of PH $\downarrow$. Annals of Pure and Applied Logic. Vol. 104 July 2000. pp. 279-303.

[14] C. Pollett. Nepomnjaščiì's Theorem and Independence Proofs in Bounded Arithmetic. Electronic Colloquium on Computational Complexity. TR02-051.

[15] C. Pollett. $S_{k, \text { exp }}$ does not prove $N P=c o-N P$ uniformly. Submitted.

[16] C. Pollett and R. Pruim. Strengths and Weaknesses of LH Arithmetic. Mathematical Logic Quarterly. 48:221-243(No.2) Feb. 2002.

[17] C. Pollett. Structure and definability in general bounded arithmetic theories. Annals of Pure and Applied Logic. Vol. 100. pages 189-245, October 1999.

[18] G. Takeuti. RSUV isomorphisms. In P. Clote and J. Krajíček, editors, Arithmetic, Proof Theory and Computational Complexity, volume 23 of Oxford Logic Guides, pages 364-386. Clarendon Press, Oxford, 1993.

[19] G. Takeuti. Frege Proof Systems and $T N C^{0}$. The Journal of Symbolic Logic, 63(2):709-738, June 1998.

[20] C. Wrathall. Rudimentary Predicates and Relative Computation. SIAM Journal of Computing, Vol. 7 pp. 194-209, 1978.

[21] D. Zambella. End Extensions of Models of Linearly Bounded Arithmetic. Annals of Pure and Applied Logic 88(2-3):263-277, 1997. 